Research Article

\title{
Salivary Protein Profile and Food Intake: A Dietary Pattern Analysis
}

\author{
Teresa Louro, ${ }^{1}$ Carla Simões, ${ }^{1}$ Wilmara Lima, ${ }^{2}$ Laura Carreira, ${ }^{2}$ Paula Midori Castelo $\left({ }^{1},{ }^{3}\right.$ \\ Henrique Luis $\mathbb{D}^{4},{ }^{4,5}$ Pedro Moreira $\mathbb{D}^{6},{ }^{6}$ and Elsa Lamy $\mathbb{D}^{1}$
}

${ }^{1}$ MED (Mediterranean Institute for Agriculture, Environment and Development), Institute for Advanced Studies and Research (IIFA)IIFA-Instituto de Investigação e Formação Avançada, University of Évora, Évora, Portugal

${ }^{2}$ Student from the Department of Chemistry, University of Évora, Évora, Portugal

${ }^{3}$ Dept. Pharmaceutical Sciences, Universidade Federal de São Paulo (UNIFESP), Diadema, Brazil

${ }^{4}$ School of Dental Medicine, University of Lisbon, Research Unit of Oral and Biomedical Sciences (UICOB), Lisbon, Portugal

${ }^{5}$ Center for Innovative Care and Health Technology (ciThecCare), Politécnico de Leiria, Leiria, Portugal

${ }^{6}$ Faculdade de Ciências da Nutrição e Alimentação da Universidade Do Porto, Porto 4150-180, Portugal

Correspondence should be addressed to Elsa Lamy; ecsl@uevora.pt

Received 7 October 2020; Revised 25 March 2021; Accepted 30 March 2021; Published 16 April 2021

Academic Editor: Roxana Valdés-Ramos

Copyright (c) 2021 Teresa Louro et al. This is an open access article distributed under the Creative Commons Attribution License, which permits unrestricted use, distribution, and reproduction in any medium, provided the original work is properly cited.

\begin{abstract}
Saliva research has gained interest due to its potential as a source of biomarkers. One of the factors inducing changes in saliva, in the short term, is food intake, and evidence exist about changes in salivary proteome induced by some food components. Since this topic of research is in its early stages, it was hypothesized that saliva protein composition could be associated with different levels of adherence to dietary patterns that contain higher amounts of plant products. The aim of the present study was to test this hypothesis, in adults, by comparing salivary protein electrophoretic profiles of individuals with different diet characteristics, particularly dietary patterns (DP) that exhibit different proportions of animal and plant-based products. Dietary habits were assessed in 122 adults (61 from each sex, with ages ranging from 20 to 59 years) using Food Frequency Questionnaires. To identify the dietary patterns, a principal component analysis was used. Individual's non-stimulated saliva was evaluated for flow rate, $\mathrm{pH}$, protein concentration, $\alpha$-amylase activity, and electrophoretic protein profiles. Seven dietary patterns (DP) were identified. Salivary amylase enzymatic activity was positively associated with animal-based and starchy foods DP, and with plant-based fatty foods without wine DP. At the same time, protein bands containing amylase and type S cystatins were positively associated with the cheese/yoghurt and wine DP. Our results support the association of salivary proteomics and different dietary patterns and highlight the need of considering food consumption habits in studies using saliva, since this is a factor associated with variations in the composition of this fluid.
\end{abstract}

\section{Introduction}

Nowadays, due to global climate changes and the rise of food-related diseases (e.g., obesity, cardiovascular diseases, etc.), dietary patterns are becoming a major issue. Different methodologies are used to access dietary habits, most of which rely on subjective reports by individuals, which implies engagement and memory capacities [1], which may result in increased error in the data obtained. Moreover, individuals often misreport dietary intake, expressing what they believe to be adequate eating amounts, this misreport being even more frequent for people with a history of dieting and being overweight $[2,3]$. For this reason, objective biomarkers identification continues to be important to increase accuracy of dietary intake assessment. Some biological markers have been tentatively assessed, with reports of urinary biomarkers of total protein [4], coffee and tea [5] or garlic intake [6], as well as plasmatic biomarkers of fruit and vegetable intake [7], among others, having been used. 
Saliva study gained interest due to the potential of this fluid as a non-invasive and stress-free source of biomarkers for different pathologies and physiological conditions $[8,9]$. In the nutrition field of study, the relationship between saliva and diet starts now to be studied (e.g., [10]) but many aspects remain to be understood. There are evidences that saliva participates in both oral processing and food sensory perception (e.g., [11-13]). Moreover, salivary gland secretion responds to diet, representing a potential source for intake biomarkers that needs to be explored. In animals, it was observed that species having different feeding niches differ in their salivary proteomes $[14,15]$. Tannins (a type of polyphenols) are the major dietary compounds linked to the salivary differences that these animal species present, and it is possible that these differences are due to the tannin amounts in food. In fact, changes in salivary glands and in the amounts of particular salivary proteins (e.g., proline-rich proteins and alpha amylase) have been linked to the proportion of polyphenols/tannins in diet (e.g., [16-18]). From our knowledge, for humans, limited information exists about potential variations in saliva composition according to polyphenol intake levels, although the perception of astringency induced by these compounds has been demonstrated to be related to saliva composition [19]. Another nutritional compound potentially linked to saliva composition is starch. Variations in starch levels in diet was proposed to be related to differences in amylase gene (Amy1) copy number variants, with higher number of copies in populations with diets rich in starch [20, 21]. Additionally, short-term changes in salivary proteome were observed for bread ingestion [22, 23].

According to what was stated above, it is possible to hypothesize that saliva protein composition can vary among adults with different dietary patterns. Moreover, a relationship between saliva and diet composition was recently proposed, in studies performed in children [10, 24], reinforcing this hypothesis.

The objective of this work was to evaluate the potential of saliva as a non-invasive and objective tool for assessing potential association with dietary intake in adults, which may be of valuable use, particularly in new epidemiological studies.

\section{Material and Methods}

2.1. Participants. A convenience sample of 122 participants (61 from each sex), 20 to 59 years old, were recruited from North Alentejo region of Portugal, which is an interior region from the country characterized by a close link to agriculture. Participants were randomly recruited from all the municipalities of the referred region, since some variability in food habits may exist between cities and villages. This allowed us to have higher representativity of the region, although comparisons between dietary habits between different regions were not the aim of the present study. Only participants without self-reported and visible signs of oral (e.g., caries) or nasal health problems (e.g., smell loss, obstruction, etc.) participated in this study. On the data collection day, participants were asked to arrive at test room between 10:00 am. and 11:00 am., at least $1 \mathrm{~h} 30 \mathrm{~m}$ after breakfast intake. Before the beginning of the study, all subjects read and signed an informed consent form. All procedures were performed according to the Declaration of Helsinki for Medical Research Involving Human Subjects and had ethical approval from the Ethical Committee of the University of Evora.

2.2. Anthropometric Data Collection. Due to the potential association between Body Mass Index (BMI) and saliva composition $[13,25]$, height and weight were assessed and measured according to the European Health Examination Survey procedures [26]. Since the relationship between food intake and obesity was not a major aim of this study, only BMI was assessed (rather than other anthropometric measures) for controlling a possible effect in saliva composition. The participants were in a stand position wearing light cloths and barefoot. A portable stadiometer (Seca 214) and a digital scale accurate to the nearest $0.1 \mathrm{~kg}$ (Seca 803) were used to access height and weight, respectively. BMI was calculated by dividing the weight $(\mathrm{kg})$ by the square of the height $\left(\mathrm{m}^{2}\right)$. Normal weight, pre-obese, and obese were considered for the respective values: $18.5<\mathrm{BMI}<25,25<\mathrm{BMI}<30$, and $\mathrm{BMI}>30$ [27]. Since only one individual presented a BMI lower than 18.5, she was considered in normal weight group.

2.3. Food Intake Assessment. Participants were asked to report their frequency of food consumption by completing a self-administered, semi-quantitative food frequency questionnaire (FFQ), validated for Portuguese adults [28]. Before questionnaire completion, the researcher responsible for data collection explained how the questionnaires should be filled. The FFQ is an 86-item questionnaire, that includes individual food items and/or food groups and beverages and assesses usual dietary intake frequency over the previous 12 months. A blank space to introduce other foods, not correspondent to any of the 86 items, was provided. In that blank space, participants wrote the food name, portion, and intake frequency, using the same frequency possibilities. Food intake was calculated by multiply one of the nine possibilities of frequency of consumption (from "never or less than once per month" to "six or more times a day"), by the weight of the standard portion size of the food item, resulting in the daily intake of each food item. Participants were instructed to fill the questionnaire taking into consideration the food portion indicated. For example, if meat is consumed one time per day, in an amount representing the double of the portion indicated, then participant filled as consuming 2 times per day. Energy and nutritional intake were estimated using an adapted Portuguese version of the nutritional analysis software Food Processor Plus (ESHA Research Inc., Salem, OR, USA). When other foods or culinary food preparations, not contained in the 86 items of the FFQ, were reported, their nutritional value was estimated and added to capture the person's total nutrient intake.

For further constitution of dietary patterns, foods with similar nutritional characteristics were grouped, according to a previous study [29], resulting in 29 food/food groups 
that were further used in a multivariate analysis, as further described. The daily intake of each food group was obtained by summing the individual daily intakes of the foods that constituted it (for example, the daily intake of fresh fruit represents the sum of individual fruits daily intakes). Since that previous work was in children [29] and did not consider alcoholic beverages in that age group, we added wine and alcoholic beverages different from wine as two variables to include in the constitution of the dietary patterns.

2.4. Saliva Collection and Cleaning. Saliva collection occurred with a minimum of $1 \mathrm{~h} 30 \mathrm{~m}$ from the last food or beverage intake (breakfast), to avoid any influence of previous ingestion in salivary protein composition. Before saliva collection, individuals were asked to rinse with water to remove any food debris and "old" saliva. Saliva was collected without stimulation. Participants were requested to accumulate all saliva produced in the mouth and spitting it to a tube, maintained on ice, each time they need it. This collection occurred for a period of 3 minutes. Saliva was maintained on ice until laboratory arrival, where it was stored for one day at $-20^{\circ} \mathrm{C}$. To remove mucins and cell and/ or food residues, saliva samples were thawed on ice and centrifuged at $13,000 \mathrm{~g}$ for 30 minutes at $4^{\circ} \mathrm{C}$. The supernatant was recovered and stored at $-80^{\circ} \mathrm{C}$ until subsequent analysis.

\subsection{Saliva Composition Analysis}

2.5.1. Saliva Flow Rate, $p H$, and Total Protein Concentration. Saliva flow rate was assessed by assuming that saliva density is 1.0. Tubes containing saliva were weighed, with the weight of the empty tube being subtracted. The final value was divided by the number of minutes during which saliva was collected. The $\mathrm{pH}$ of saliva samples was measured using a calibrated $\mathrm{pH}$ meter (Hanna Instruments) and recording to two decimal places. Total protein concentration was determined by the Bradford method, using bovine serum albumin (BSA) as standard, and plates were read at $600 \mathrm{~nm}$ in a microplate reader (Glomax, Promega).

2.5.2. SDS-PAGE Separation and Protein Profile Analysis. Each saliva sample was run in duplicate. For each sample, a volume corresponding to $7.5 \mu \mathrm{g}$ total protein was mixed with sample buffer and run on each lane of a $14 \%$ polyacrylamide mini-gel (Protean xi, Bio-Rad, CA, USA) using a Laemmli buffer system, as described elsewhere [30]. Each electrophoretic run was performed at a constant voltage of $140 \mathrm{~V}$ until front dye reached the end of the gel. Gels were fixed for 1 hour in $40 \%$ methanol $/ 10 \%$ acetic acid, followed by staining for 2 hours with Coomassie Brilliant Blue (CBB) G-250. Gel images were acquired using a scanning Molecular Dynamics densitometer with internal calibration and LabScan software (GE Healthcare), and images were analysed using GelAnalyzer software (GelAnalyzer 2010a by Istvan Lazar, http://www.gelanalyzer.com) for the volume percentage of each protein band. Molecular masses were determined in accordance with molecular mass standards (Bio-Rad Precision Plus Protein Dual Colour 161-0394) run with protein samples. The identification of the proteins contained in the bands observed in SDS-PAGE salivary profiles was based on previous data [22].

\subsubsection{Salivary Amylase Enzymatic Activity. A Salimetrics ${ }^{\circledR}$} kit was used to determine the enzymatic activity of salivary amylase according to the manufacturer's recommendations. Briefly, saliva samples were diluted $200 \times$ and applied on the microplate in duplicate, followed by application of a substrate (2-chloro-p-nitrophenol) preheated to $37^{\circ} \mathrm{C}$. The mixture was incubated at $37^{\circ} \mathrm{C}$ for 1 minute, and absorbance values were read at $405 \mathrm{~nm}$ in a plate reader spectrophotometer, followed by incubation for an additional 2 minutes at $37^{\circ} \mathrm{C}$ and a new reading at $405 \mathrm{~nm}$. The enzymatic activity of amylase $(\mathrm{U} / \mathrm{ml})$ was calculated by the following formula: $(\triangle \mathrm{Abs} . / \mathrm{min} \times \mathrm{TV} \times \mathrm{DF}) /(\mathrm{MMA} \times \mathrm{SV} \times \mathrm{LP})$, where $\Delta \mathrm{Abs} . /$ Min is absorbance variation per minute, TV is total test volume $(0.287 \mathrm{~mL}), \mathrm{DF}$ is dilution factor, MMA is millimolar absorbance of substrate 2-chloro-p-nitrophenol (12.9), SV is sample volume $(0.007 \mathrm{~mL})$, and LP is light path (0.97, specific for plate received with kit).

2.6. Statistical Analysis. The values of total protein concentration, salivary secretion rate, protein band amount (volume percentage), and salivary amylase enzymatic activity were analysed statistically. Descriptive statistics was performed, and normality and homoscedasticity were tested through Shapiro-Wilk and Levene tests, respectively. Continuous variables were compared between sexes using Student's $t$-test when assumptions were fulfilled and using Mann-Whitney when not. For BMI classes (normal weigh, pre-obese and obese) comparison, one-way ANOVA or the non-parametric Kruskal-Wallis test was used, considering a confidence interval of $95 \%$.

To identify dietary patterns within the study population, multivariate statistical techniques were used. The 29 foods/ food groups constituted from the 86 items of the FFQ, as described earlier and according to a previous study [29], were considered together with 2 additional groups, namely, wine, and alcoholic beverages excluding wine. These 31 variables (amount of food consumed per day for each food/ food group) were reduced through principal component analysis (PCA). PCA with orthogonal rotation (Varimax) was used to estimate the latent factors emerging and to obtain optimal non-correlated components. The number of components (dietary patterns) to be retained was decided based on the observation of correlation matrix, on the total of variance explained by the components and on Kaiser-Meyer-Olkin (KMO) measure of sampling adequacy values above $0.600[31,32]$.

With the aim of explaining $\alpha$-amylase enzymatic activity and salivary protein profile variation (namely, the expression levels of each SDS-PAGE protein band) as dependent variables, each dietary pattern extracted from the PCA analysis was used as independent variable in the regression model, adjusting for age, BMI, sex, and total energy consumed. A 
stepwise backward process was used to obtain the final model after looking for changes in adjusted $R 2$ and $F$ values for each retained variable; the assumptions of collinearity (VIF and tolerance), non-dependent errors (Durbin-Watson), and homoscedasticity (residual analysis) were also considered.

\section{Results}

3.1. Participants Characteristics and Dietary Patterns. Forty percent of the individuals participating in this study were normal weight and $60 \%$ overweight (36\% pre-obese $+24 \%$ obese). When comparing the sexes, men presented statistically significantly higher BMI than women (Table 1), with $67.7 \%$ of men being overweight (38.7\% pre-obese $+29.0 \%$ obese) whereas $29.8 \%$ of women being pre-obese and $17.5 \%$ obese (e.g., a total of $47.3 \%$ overweight women).

When participants were analysed for their food intake, assessed by the FFQ, some statistically significant differences were observed between men and women in the energy contribution of protein (higher in women) and in the consumption of some food groups (Table 1). Women have significant higher consumption of vegetables, vegetable soup, yoghurts, and crackers/cookies with approximately $20 \%$ sugar or less, as well as white meat and lower consumption levels of canned fruit, red meat, processed meat, and alcoholic drinks. No significant differences between sexes were found in relation to mean total energy or total macronutrient intake.

It was observed a tendency for older individuals to have higher BMI. Despite no significant differences in total energy or total macronutrient intake, for foods like vegetable soup, vegetable oil, and starchy-rich foods, the daily intake was observed to be higher in obese individuals (Table 1).

To estimate the dietary patterns from the food/food groups considered [29], PCA was performed as described in material and methods section. PCA adequacy was evaluated before analysis. Matrix component inspection allowed choosing the variables (food/food groups) with coefficients higher than 0.30 in at least one component, resulting in 24 variables (food/food groups) including the final model. The general measure of KMO was 0.606, and Bartlett sphericity was statistically significant $(p<0.0001)$.

It was possible to obtain 7 components, which explained $59 \%$ of total variance (Table 2), corresponding to the following dietary patterns: DP1 with positive loadings for vegetables and soup, olives, fresh fruit, bread and starch-rich foods, and sugar sweetened beverages (SSB) (plant-based with SSB DP); DP2 presented positive loadings for fish, meat, eggs, and starch-rich foods (animal-based and starchy foods pattern); DP3 presented high positive scores for fast food, processed foods, and SSB (fast-food pattern); DP4 presented positive loadings for olive oil, margarine, and butter, as well as for pastry and negative loadings for pulses (dietary fats and pastry pattern); DP5 presented positive loadings for dairy desserts, pastry, canned fruits, and readyto-eat cereals (sweet foods pattern); DP6 presented positive loadings for olives and nuts, but negative loadings for wine (plant-based fatty foods without wine pattern); and DP7 presented positive loadings for yoghurt, cheese, and wine (cheese/yoghurt and wine pattern).

3.2. Saliva Composition. SDS-PAGE electrophoresis allowed the consistent separation and comparison of well resolved 12 protein bands, with apparent molecular masses between 14.1 and $88.0 \mathrm{kDa}$ (Figure 1), containing proteins previously identified [22] (Table 3).

By comparing the sexes for saliva composition, men presented higher expression levels of the protein bands $G$ $(4.39 \pm 0.73$ vs. $3.98 \pm 0.85$, for men and women, respectively; $p=.005)$ and $\mathrm{J}(8.54 \pm 2.74$ vs. $7.41 \pm 2.14$, for men and women, respectively; $p=.014)$.

3.3. Dietary Patterns and Saliva Composition. The significant associations found for linear multiple regression models between salivary parameters, namely, amylase enzymatic activity, protein bands $\mathrm{E}$ (identified as amylase) and $\mathrm{J}$ (identified as cystatins), and dietary patterns, are shown in Table 4. The protein bands A, B, I1, I2, and K did not show significant associations, and protein bands $\mathrm{C}, \mathrm{D}$, and $\mathrm{G}$ were only associated with age (positively), BMI (positively), and sex (high in men), respectively.

Looking at the linear multiple regression models between dietary patterns and salivary parameters, adjusting for confounders (sex, age, BMI, and energy intake), in Table 4, it was possible to observe that DP7 (fermented dairy and wine pattern) was positively associated with bands E (containing amylase) and J (containing S-type cystatins), and DP6 (plant-based fatty foods without wine pattern) and DP2 (animal-based and starchy foods pattern) were positively associated with amylase enzymatic activity.

\section{Discussion}

In the present study, PCA analysis was used to identify combinations of foods, named as dietary patterns and which constituted not-correlated variables, used as independent variables in the regression models. As such, these dietary patterns do not represent groups of individuals with particular food consumption patterns.

Dietary patterns constituted by high proportion of plantbased fatty foods (without wine, DP6) together with dietary patterns based on foods of animal origin and starch-rich foods (DP2) were associated with the salivary amylase enzymatic activity. This is in line with other studies associating salivary amylase with starchy foods intake [21] although, to the best of our knowledge, no previous studies related salivary amylase with olives and nuts intake. The different results obtained when amylase was considered in terms expression levels, comparatively to when it was considered in terms of its enzymatic activity, are not surprising, since a lack of association between the enzymatic activity of this protein and the expression levels of its different proteoforms has been previously reported [33].

In relation to a dietary pattern with high contribution of fermented dairy and wine (DP7), an association with the 
TABLE 1: Sample characteristics, nutritional, and food intake differences between sexes and BMI (values are mean \pm standard deviation $(\mathrm{SD}))$.

\begin{tabular}{|c|c|c|c|c|c|c|c|}
\hline Parameters & Men & Women & $\begin{array}{c}p \text {-value } \\
\text { (sex) }\end{array}$ & NW & Pre-obese & Obese & $\begin{array}{c}p \text {-value } \\
\text { (BMI) }\end{array}$ \\
\hline Age (years) & $40.7 \pm 10.9$ & $38.3 \pm 10.7$ & 0.477 & $37.1 \pm 10.9$ & $41.5 \pm 1.7$ & $42.6 \pm 9.6$ & .054 \\
\hline BMI $\left(\mathrm{kg} / \mathrm{m}^{2}\right)$ & $27.9 \pm 4.2$ & $25.9 \pm 5.2$ & $0.014^{*}$ & - & - & - & \\
\hline \multicolumn{8}{|l|}{ Nutritional parameters } \\
\hline Energy (kcal/day) & $2346 \pm 746$ & $2280 \pm 734$ & 0.627 & $2304 \pm 723$ & $2361 \pm 787$ & $2211 \pm 538$ & 0.650 \\
\hline Protein (g/day) & $102.8 \pm 37.6$ & $107.8 \pm 35.6$ & 0.457 & $103.1 \pm 36.1$ & $105.5 \pm 35.7$ & $106.5 \pm 32.1$ & 0.924 \\
\hline Protein $(\% \mathrm{TEV})$ & $17.6 \pm 3.9$ & $19.2 \pm 3.7$ & $0.027^{*}$ & $18.1 \pm 4.0$ & $18.1 \pm 3.3$ & $19.4 \pm 4.3$ & 0.198 \\
\hline Total carbohydrate (g/day) & $263.4 \pm 101.7$ & $256.1 \pm 92.2$ & 0.911 & $265.3 \pm 95.0$ & $268.4 \pm 106.5$ & $236.8 \pm 75.0$ & 0.472 \\
\hline Total carbohydrate (\% TEV) & $44.9 \pm 10.0$ & $44.9 \pm 8.8$ & 0.976 & $46.3 \pm 11.0$ & $45.4 \pm 7.0$ & $42.4 \pm 8.1$ & 0.143 \\
\hline Total sugars (g/day) & $119.0 \pm 58.5$ & $113.5 \pm 51.4$ & 0.853 & $124.9 \pm 60.5$ & $111.5 \pm 47.7$ & $103.6 \pm 44.6$ & 0.351 \\
\hline Total sugars (\% TEV) & $20.5 \pm 7.8$ & $19.9 \pm 6.4$ & 0.651 & $21.6 \pm 8.6$ & $19.4 \pm 4.9$ & $18.5 \pm 5.9$ & 0.226 \\
\hline SFA (g/day) & $26.6 \pm 11.0$ & $26.3 \pm 9.5$ & 0.951 & $26.7 \pm 10.1$ & $26.8 \pm 11.4$ & $25.6 \pm 8.9$ & 0.866 \\
\hline SFA (\% TEV) & $10.3 \pm 2.6$ & $10.5 \pm 2.3$ & 0.684 & $10.5 \pm 2.5$ & $10.2 \pm 2.4$ & $10.5 \pm 2.2$ & 0.949 \\
\hline MUFA (g/day) & $41.2 \pm 16.9$ & $42.9 \pm 17.8$ & 0.535 & $40.2 \pm 16.3$ & $43.0 \pm 17.9$ & $41.4 \pm 13.9$ & 0.685 \\
\hline MUFA (\% TEV) & $15.8 \pm 4.0$ & $17.1 \pm 4.0$ & 0.072 & $16.0 \pm 4.0$ & $16.3 \pm 3.9$ & $16.8 \pm 3.3$ & 0.458 \\
\hline PUFA (g/day) & $15.8 \pm 6.0$ & $15.8 \pm 6.4$ & 0.901 & $14.6 \pm 5.7$ & $16.6 \pm 6.2$ & $15.9 \pm 5.8$ & 0.137 \\
\hline PUFA (\% TEV) & $6.1 \pm 1.5$ & $6.3 \pm 1.4$ & 0.351 & $5.9 \pm 1.5$ & $6.3 \pm 1.3$ & $6.4 \pm 1.3$ & 0.106 \\
\hline Cholesterol (mg/day) & $361.3 \pm 167.7$ & $345.6 \pm 134.0$ & 0.578 & $345.0 \pm 148.3$ & $358.6 \pm 165.9$ & $369.3 \pm 141.7$ & 0.774 \\
\hline Fibre (g/day) & $26.2 \pm 13.0$ & $28.4 \pm 12.9$ & 0.206 & $27.8 \pm 13.0$ & $28.5 \pm 13.4$ & $25.7 \pm 12.5$ & 0.695 \\
\hline \multicolumn{8}{|l|}{ Foods/food groups (g/day) ${ }^{\#}$} \\
\hline Vegetables & $387.9 \pm 268.0$ & $499.7 \pm 255.4$ & $0.038^{*}$ & $434.9 \pm 283.1$ & $424.4 \pm 230.6$ & $543.4 \pm 265.5$ & 0.106 \\
\hline Vegetable soup & $194.5 \pm 167.4$ & $272.0 \pm 154.6$ & $0.003^{*}$ & $247.6 \pm 237.2$ & $206.0 \pm 130.1$ & $298.0 \pm 136.9$ & $0.016^{*}$ \\
\hline Fresh fruits & $253.8 \pm 177.1$ & $353.6 \pm 239.3$ & $0.027^{*}$ & $348.6 \pm 270.3$ & $304.6 \pm 196.8$ & $255.1 \pm 188.3$ & 0.281 \\
\hline Canned fruits & $10.7 \pm 21.2$ & $5.6 \pm 12.8$ & $0.006^{*}$ & $6.4 \pm 13.5$ & $9.3 \pm 23.5$ & $7.8 \pm 11.8$ & 0.300 \\
\hline Olives & $8.1 \pm 15.4$ & $13.4 \pm 36.6$ & 0.230 & $14.3 \pm 38.9$ & $8.9 \pm 18.2$ & $7.7 \pm 12.3$ & 0.719 \\
\hline Nuts & $10.6 \pm 15.0$ & $17.2 \pm 22.9$ & 0.193 & $13.9 \pm 29.9$ & $15.5 \pm 19.3$ & $17.2 \pm 23.2$ & 0.223 \\
\hline Fish & $72.7 \pm 46.3$ & $82.9 \pm 6.4$ & 0.168 & $68.5 \pm 43.6$ & $82.7 \pm 48.2$ & $89.6 \pm 55.6$ & 0.156 \\
\hline White meat & $55.1 \pm 47.0$ & $72.9 \pm 47.7$ & $0.039^{*}$ & & & & \\
\hline Red meat & $43.0 \pm 34.3$ & $30.8 \pm 27.3$ & $0.032^{*}$ & $32.7 \pm 27.8$ & $38.1 \pm 35.4$ & $38.8 \pm 27.9$ & 0.572 \\
\hline Processed meat ${ }^{1}$ & $31.9 \pm 40.5$ & $17.5 \pm 14.3$ & $0.012^{*}$ & $23.8 \pm 34.4$ & $24.5 \pm 35.0$ & $24.6 \pm 17.1$ & 0.347 \\
\hline Eggs & $14.1 \pm 11.1$ & $14.7 \pm 9.8$ & 0.397 & $12.9 \pm 9.5$ & $15.4 \pm 11.6$ & $15.7 \pm 10.5$ & 0.325 \\
\hline Olive oil & $10.9 \pm 9.5$ & $12.9 \pm 9.1$ & 0.084 & $11.4 \pm 10.6$ & $14.0 \pm 12.9$ & $11.6 \pm 9.1$ & 0.868 \\
\hline Vegetable oil & $2.1 \pm 3.5$ & $1.5 \pm 3.1$ & 0.213 & $1.0 \pm 2.4$ & $2.3 \pm 3.3$ & $2.0 \pm 3.8$ & $0.006^{*}$ \\
\hline Butter & $2.5 \pm 2.2$ & $3.5 \pm 3.0$ & 0.059 & $2.9 \pm 2.3$ & $2.9 \pm 2.7$ & $2.9 \pm 3.1$ & 0.843 \\
\hline Margarine & $1.2 \pm 1.6$ & $1.3 \pm 1.6$ & 0.438 & $1.7 \pm 2.2$ & $1.5 \pm 2.3$ & $1.2 \pm 2.4$ & 0.366 \\
\hline Milk & $167.7 \pm 164.4$ & $231.5 \pm 206.7$ & 0.114 & $199.4 \pm 212.4$ & $216.4 \pm 179.4$ & $186.9 \pm 152.5$ & 0.524 \\
\hline Yoghurt & $62.7 \pm 85.3$ & $93.6 \pm 90.6$ & $0.009^{*}$ & $84.6 \pm 99.9$ & $60.4 \pm 72.6$ & $86.0 \pm 90.9$ & 0.399 \\
\hline Cheese & $18.1 \pm 21.3$ & $15.7 \pm 16.3$ & 0.889 & $16.7 \pm 19.3$ & $16.5 \pm 19.2$ & $19.0 \pm 19.2$ & 0.623 \\
\hline Milk-based puddings & $13.9 \pm 16.6$ & $9.2 \pm 9.2$ & 0.323 & $11.1 \pm 13.2$ & $12.0 \pm 15.3$ & $10.2 \pm 10.7$ & 0.982 \\
\hline Ice-cream & $7.5 \pm 8.7$ & $5.6 \pm 8.1$ & 0.083 & $6.3 \pm 8.4$ & $7.9 \pm 10.4$ & $4.9 \pm 4.7$ & 0.791 \\
\hline Starch-rich foods (rice, potatoes, pasta) ${ }^{2}$ & $136.8 \pm 72.0$ & $136.4 \pm 71.1$ & 1.000 & $120.1 \pm 66.7$ & $156.5 \pm 53.8$ & $153.8 \pm 109.4$ & $0.021^{*}$ \\
\hline Bread & $63.8 \pm 51.7$ & $77.0 \pm 53.7$ & 0.110 & $76.6 \pm 54.7$ & $75.1 \pm 57.1$ & $59.4 \pm 39.7$ & 0.542 \\
\hline Ready-to-eat cereals & $11.6 \pm 14.8$ & $9.3 \pm 14.1$ & 0.306 & $9.5 \pm 14.2$ & $12.5 \pm 15.3$ & $7.5 \pm 12.9$ & 0.357 \\
\hline Sugar sweetened beverages $(\mathrm{SSB})^{3}$ & $170.1 \pm 216.3$ & $84.6 \pm 100.2$ & 0.100 & $134.6 \pm 192.4$ & $107.2 \pm 145.3$ & $128.1 \pm 180.6$ & 0.839 \\
\hline Coffee & $76.5 \pm 57.6$ & $63.4 \pm 49.5$ & 0.330 & $64.5 \pm 56.6$ & $74.5 \pm 49.5$ & $78.8 \pm 50.7$ & 0.585 \\
\hline Tea & $32.4 \pm 58.6$ & $68.3 \pm 88.3$ & 0.246 & $42.2 \pm 79.7$ & $45.2 \pm 74.0$ & $43.1 \pm 72.1$ & 0.994 \\
\hline Fast-food ${ }^{4}$ & $37.8 \pm 27.8$ & $29.3 \pm 15.4$ & 0.163 & $32.7 \pm 23.8$ & $32.1 \pm 20.3$ & $37.5 \pm 33.8$ & 0.936 \\
\hline Pastry & $40.1 \pm 36.4$ & $30.6 \pm 25.7$ & 0.245 & $36.0 \pm 31.0$ & $40.3 \pm 38.1$ & $25.7 \pm 22.7$ & 0.237 \\
\hline Crackers/cookies ${ }^{5}$ & $5.5 \pm 7.6$ & $10.9 \pm 13.5$ & $0.029^{*}$ & $9.8 \pm 12.4$ & $7.7 \pm 10.4$ & $6.8 \pm 9.9$ & 0.433 \\
\hline Pulses & $51.9 \pm 57.6$ & $35.5 \pm 36.7$ & 0.498 & $44.2 \pm 49.9$ & $46.6 \pm 50.1$ & $42.9 \pm 45.6$ & 0.975 \\
\hline Wine & $36.6 \pm 41.3$ & $12.2 \pm 25.3$ & $<0.001^{*}$ & $17.4 \pm 30.8$ & $25.4 \pm 36.9$ & $38.0 \pm 42.6$ & 0.072 \\
\hline Alcoholic beverages (apart from wine) ${ }^{6}$ & $61.9 \pm 74.5$ & $14.1 \pm 30.4$ & $<0.001^{*}$ & $26.1 \pm 43.6$ & $43.1 \pm 63.2$ & $52.1 \pm 83.6$ & 0.173 \\
\hline
\end{tabular}


TABLE 2: Component loadings obtained by principal component analysis with Varimax rotation. Each component was named as "dietary pattern."

\begin{tabular}{|c|c|c|c|c|c|c|c|}
\hline \multirow[b]{2}{*}{$\%$ Var explained } & \multicolumn{7}{|c|}{ Component } \\
\hline & $\begin{array}{c}1 \\
16.0 \\
\end{array}$ & $\begin{array}{c}2 \\
11.0 \\
\end{array}$ & $\begin{array}{c}3 \\
8.7 \\
\end{array}$ & $\begin{array}{c}4 \\
6.9 \\
\end{array}$ & $\begin{array}{c}5 \\
5.7 \\
\end{array}$ & $\begin{array}{c}6 \\
5.4 \\
\end{array}$ & $\begin{array}{c}7 \\
5.0 \\
\end{array}$ \\
\hline Yoghurt & & & & & & & 0.422 \\
\hline Cheese & & & & & & & 0.741 \\
\hline Milk-based puddings & & & & & 0.599 & & \\
\hline Eggs & & 0.637 & & & & & \\
\hline Meat & & 0.765 & & & & & \\
\hline Processed meat & & & 0.575 & & & & \\
\hline Fish & & 0.823 & & & & & \\
\hline Olive oil & & & & 0.665 & & & \\
\hline Margarine & & & & 0.633 & & & \\
\hline Butter & & & & 0.740 & & & \\
\hline Bread & 0.779 & & & & & & \\
\hline Ready-to-eat cereals & & & & & 0.584 & & \\
\hline Starch-rich foods (rice, potatoes, pasta) & 0.456 & 0.471 & & & & & \\
\hline Pastry & & & & 0.486 & 0.472 & & \\
\hline Vegetables & 0.725 & & & & & & \\
\hline Pulses & & & & -0.418 & & & \\
\hline Fresh fruits & 0.521 & & & & & & \\
\hline Canned fruits & & & & & 0.727 & & \\
\hline Nuts & & & & & & 0.732 & \\
\hline Olives & 0.449 & & & & & 0.667 & \\
\hline $\mathrm{SSB}^{1}$ & 0.467 & & 0.637 & & & & \\
\hline Fast-food & & & 0.778 & & & & \\
\hline Vegetable soup & 0.712 & & & & & & \\
\hline Wine & & & & & & -0.518 & 0.423 \\
\hline
\end{tabular}

Rotation converted with 16 interactions. Values lower than 0.40 were omitted. ${ }^{1}$ Sugar sweetened beverages.

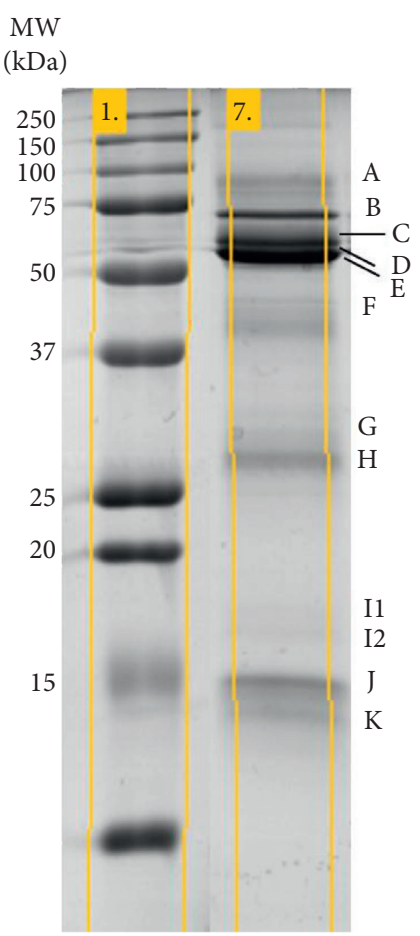

FIGURE 1: Representative SDS-PAGE salivary profile showing the 12 protein bands consistently present in the different individuals studied (letters on the right side); MW, molecular mass $(\mathrm{kDa})$. expression level of one band containing $\alpha$-amylase and one band containing S-type cystatins was observed. Concerning wine, it may be a source of tannins, which are known to affect saliva proteome, in animal models [16, 34]. In fact, in mice, it was previously observed that increased levels of polyphenols (tannins) in the diet result in increased expression levels of salivary amylase bands, in SDS-PAGE profiles [18]. Concerning cystatins, these are inhibitors of cysteine proteases, the family of S-type cystatins being secreted by salivary glands. Different studies show a relationship between salivary cystatins and the bitterness or astringency (e.g., $[13,35,36]$ ) that is associated with the polyphenol content of foods and beverages, such as wines (particularly red wines). If higher levels of salivary S-type cystatins are present in individuals with low sensitivity to bitterness and astringency, as reported by some authors [11], it can be hypothesized that the positive association between S-type cystatins and DP7 results from the lower intensity with which these oral sensations are perceived and consequently the higher acceptance/preference for wine, in consequence of salivary cystatins. At the same time, another hypothesis is that a higher intake of wine can induce higher levels of salivary S-type cystatins. This hypothesis is supported by studies showing that exposing rats to tannins or bitter compounds results in the increase of S-type salivary cystatins secretion and in the increase of bitter taste compounds acceptance $[35,37]$. Anyway, this hypothesis needs 
TABLE 3: Proteins present in the bands observed in SDS-PAGE salivary profiles.

\begin{tabular}{|c|c|c|}
\hline SDS-PAGE Band & Protein identification\# & Apparent molecular mass $(\mathrm{kDa})$ \\
\hline A & Polymeric immunoglobulin receptor & 88.0 \\
\hline $\mathrm{B}$ & Serum albumin & 71.0 \\
\hline $\mathrm{C}$ & $\alpha$-Amylase 1 & 64.0 \\
\hline $\mathrm{D}$ & $\alpha$-Amylase 1 & 58.2 \\
\hline $\mathrm{E}$ & $\alpha$-Amylase 1 & 55.0 \\
\hline $\mathrm{F}$ & $\begin{array}{l}\text { Zinc- } \alpha 2 \text {-glycoprotein } \\
\text { Carbonic anhydrase VI }\end{array}$ & 42.0 \\
\hline G & $\begin{array}{l}\text { Zymogen granule protein } 16 \text { homolog B } \\
\text { Immunoglobulin kappa constant }\end{array}$ & 31.5 \\
\hline $\mathrm{H}$ & Immunoglobulin kappa constant & 27.0 \\
\hline I1 & Prolactin inducible protein (PIP) & 16.8 \\
\hline $\mathrm{I} 2$ & Prolactin inducible protein (PIP) & 16.2 \\
\hline $\mathrm{J}$ & $\begin{array}{l}\text { Cystatin-SN } \\
\text { Cystatin-S }\end{array}$ & 14.5 \\
\hline $\mathrm{K}$ & Cystatin B & 14.1 \\
\hline
\end{tabular}

\# Protein identification based on previous studies with human saliva collected in the same conditions.

TABLE 4: Linear multiple regression models between dietary patterns and salivary parameters.

\begin{tabular}{|c|c|c|c|c|c|c|c|c|}
\hline Dependent variable & Independent variable & Coeff & CI (95\%) & $t$ & $p$ value & $F$ & $R 2$ adj & Durbin- Watson \\
\hline \multirow{6}{*}{ Band $\mathrm{E}$} & Constant & 1.390 & 0.686 to 2.095 & 3.912 & $<0.001$ & \multirow{6}{*}{2.765} & \multirow{6}{*}{0.072} & \multirow{6}{*}{1.953} \\
\hline & Dietary pattern 7 & 0.106 & 0.012 to 0.199 & 2.247 & $0.027^{*}$ & & & \\
\hline & Energy & $6.208 \mathrm{E}-6$ & 0.000 to 0.000 & 0.136 & 0.892 & & & \\
\hline & Sex & 0.023 & -0.159 to 0.204 & 0.248 & 0.804 & & & \\
\hline & Age & -0.008 & -0.017 to 0.000 & -1.868 & 0.064 & & & \\
\hline & BMI & 0.027 & 0.008 to 0.047 & 2.766 & 0.007 * & & & \\
\hline \multirow{6}{*}{ Band J } & Constant & 9.035 & 5.472 to 12.598 & 5.027 & $<0.001$ & \multirow{6}{*}{3.868} & \multirow{6}{*}{0.132} & \multirow{6}{*}{1.598} \\
\hline & Dietary pattern 7 & .691 & 0.224 to 1.157 & 2.934 & $0.004^{*}$ & & & \\
\hline & Energy & 0.000 & -0.001 to 0.000 & -0.513 & 0.609 & & & \\
\hline & Sex & -0.625 & -1.574 to .323 & -1.307 & 0.194 & & & \\
\hline & Age & 0.041 & -0.002 to 0.085 & 1.892 & 0.061 & & & \\
\hline & BMI & -0.054 & -0.151 to 0.043 & -1.099 & 0.274 & & & \\
\hline \multirow{7}{*}{$\alpha$-Amylase (U/mL) } & Constant & 167.348 & -46.456 to 381.152 & 1.553 & 0.124 & \multirow[t]{7}{*}{4.126} & 0.150 & \multirow[t]{7}{*}{1.569} \\
\hline & Dietary pattern 2 & 42.668 & 13.620 to 71.715 & 2.914 & $0.004^{*}$ & & & \\
\hline & Dietary pattern 6 & 31.012 & 2.395 to 59.629 & 2.150 & $0.034 *$ & & & \\
\hline & Energy & -0.007 & -0.037 to 0.023 & -.459 & 0.647 & & & \\
\hline & Sex & 18.932 & -36.866 to 74.730 & 0.673 & 0.502 & & & \\
\hline & Age & 4.474 & 1.905 to 7.043 & 3.456 & 0.001 * & & & \\
\hline & BMI & -5.641 & -11.433 to 0.150 & -1.933 & 0.056 & & & \\
\hline
\end{tabular}

All the models were adjusted for sex, age, BMI, and energy intake. ${ }^{*}$ Significant for $p<0.05$.

to be further tested. It is worthwhile to remember that DP7 has also a considerable contribution of fermented dairy. Although few information exists about effect of dairy in saliva composition, the levels of salivary cystatins were recently reported to increase in response to chocolate milk [38].

The association between the dietary patterns obtained by PCA analysis and saliva composition may be additionally confounded by sex, BMI, and age. As such, those parameters were included in the regression models used. Although we are unable to guarantee that the observed salivary differences between sexes are due exclusively to the sex factor, and not to the differences in the eating habits of women and men, the effect of sex in salivary proteome needs to be considered, since it has been already reported $[13,39,40]$ and reinforced in recent publications (e.g., [41]). Minor differences were observed among BMI groups in food consumption habits, although starchy foods were highly consumed by overweight and obese individuals. As for the case of sex, we cannot guarantee that the effect of BMI in saliva was not influenced by this particular difference in food intake. As such, the positive association between BMI and the band containing one amylase form (band E) can result from this higher intake of starchy foods by individuals with high BMI. But, since band $\mathrm{E}$ was not related to the patterns with starch-rich foods (DP2), other factors apart from starch consumption may explain this positive association between band $\mathrm{E}$ and obesity. Differences in saliva composition among individuals with different BMI have been already observed, which included variations in the expression levels of salivary proteins like carbonic anhydrase VI and some forms of amylase [30]. Finally, the positive association between salivary amylase 
enzymatic activity and age goes in line with previous observations about increasing salivary amylolytic activity in people with higher age [42].

\subsection{Our Study Has Some Limitations}

(1) Firstly, although in the regression model for statistical analysis, $\alpha$-amylase enzymatic activity and salivary protein profile were considered as dependent variables, while dietary patterns extracted from the PCA analysis were used as independent variables, the cross-sectional design of our study does not allow us to establish causal relationships between dietary intake and salivary parameters. Moreover, since the dietary patterns considered are, in fact, the components generated by PCA, which consist in the combinations of the different food/food groups, it is not possible to allocate each individual subject to one of these dietary patterns.

(2) Secondly, whereas the salivary parameters represent the saliva collected at one time point, the intake reported represents the frequency of the food groups consumption in the last 12 months. Short-term effect of food intake was not controlled. Moreover, it is recognized that FFQ have the limitation of being difficult to carry out to complete, and suffer from difficulties in self-estimation of portion size and biases resulting from misreporting [43]. However, the FFQ used is validated for Portuguese adults and considered as a valid tool to estimate the usual food intake in large samples. Intervention-based studies must be further done to get more controlled results about the effect of specific dietary patterns in saliva composition.

Our study has also important strengths. According to our best knowledge, this is the first study that aimed to assess how dietary habits relate to saliva protein composition. One of the main findings is that some types of salivary proteins appear to be associated with the intake of particular foods, namely, animal-based and starch-rich foods, plant-based fatty foods, fermented dairy, and wine. It is a fact that the regression models obtained presented relatively low $R^{2}$, which means that the model only explains a minor percentage of the variation in salivary proteins levels. But taking into consideration that we are working with biological models, where several other factors besides food frequency consumption can affect salivary protein composition [44, 45], this low $R^{2}$ is not surprising and the statistical significance of the model reinforces an association between salivary proteins and food habits.

\section{Conclusions}

The results obtained in the present study emphasize the potential of saliva to reflect differences in food intake. Salivary amylase and S-type cystatins are associated with dietary patterns. It is interesting to note that some combinations of foods are more related to the total enzymatic activity of amylase whereas others with the expression levels of forms of this protein. Salivary amylase and cystatins are proteins previously observed to have a link to oral food perception and more studies are necessary to confirm if they can be the result of the type of food eaten or rather the cause of food choices.

To consider salivary proteins as non-invasive biomarkers of intake, more studies are required, to define criteria, such as sex, BMI, age, and hour of the day, among others. Moreover, it will be important, in further work, to get more detailed information using other proteomic approaches. Even so, this study evidences that some of the variation in salivary protein composition, among individuals, can be related to food habits and it is important to take into account foods as a source of variation, when using saliva for studies with different purposes.

\section{Data Availability}

All the data necessary to reach the objectives of the study are included. Raw data files, which generated the results, are with the researchers.

\section{Disclosure}

The FCT-Portuguese Science Foundation was not involved in carrying out this study or submitting it for publication.

\section{Conflicts of Interest}

The authors have no conflicts of interest.

\section{Acknowledgments}

The authors would like to thank all the subjects who took part in the study. This paper was funded by the FEDER as part of the Operational Programme for Competitiveness Factors (COMPETE) and received funding from the Science and Technology Foundation (FCT) under the Project UID/ AGR/00115/2019 (ICAAM, University of Évora). Funding was additionally provided by the FCT-Portuguese Science Foundation, Research Contract CEECIND/04397/2017 to Elsa Lamy. Support was also received from the State of São Paulo Research Foundation (FAPESP), grant no. 2017/ 26400-6.

\section{References}

[1] J.-S. Shim, K. Oh, and H. C. Kim, "Dietary assessment methods in epidemiologic studies," Epidemiology and Health, vol. 22, no. 36, Article ID e2014009, 2014.

[2] J. Maurer, D. L. Taren, P. J. Teixeira et al., "The psychosocial and behavioral characteristics related to energy misreporting," Nutrition Reviews, vol. 64, no. 2, 2006.

[3] R. K. Johnson, R. P. Soultanakis, and D. E. Matthews, "Literacy and body fatness are associated with underreporting of energy intake in US low-income women using the multiplepass 24-hour recall: a doubly labeled water study," Journal of the American Dietetic Association, vol. 98, no. 10, pp. 11361140, 1998. 
[4] S. A. Bingham, "Urine nitrogen as a biomarker for the validation of dietary protein intake," Journal of Nutrition, vol. 24, pp. 921S-924S, 2003.

[5] J. A. Rothwell, F. Madrid-Gambin, M. Garcia-Aloy et al., "Biomarkers of intake for coffee, tea, and sweetened beverages," Genes \& Nutrition, vol. 15, 2018.

[6] L. Scheffler, Y. Sauermann, A. Heinlein, C. Sharapa, and A. Buettner, "Detection of volatile metabolites derived from garlic (Allium sativum) in human urine," Metabolites, vol. 6, no. 4, p. 43, 2016.

[7] M. Pennant, M. Steur, C. Moore, A. Butterworth, and L. Johnson, "Comparative validity of Vitamin $\mathrm{C}$ and carotenoids as indicators of fruit and vegetable intake: a systematic review and meta-analysis of randomised controlled trials," British Journal of Nutrition, vol. 231, pp. 14-18, 2013.

[8] E. Lamy, "Salivary proteomics in ingestive behaviour research: advances, potentialities and limitations," Journal of Integrated OMICS, vol. 8, no. 1, 2018.

[9] M. Castagnola, E. Scarano, G. C. Passali et al., "Salivary biomarkers and proteomics: future diagnostic and clinical utilities," ACTA Otorhinolaryngologica Italica, vol. 37, no. 2 , pp. 94-101, 2017.

[10] M. Morzel, C. Truntzer, E. Neyraud et al., “Associations between food consumption patterns and saliva composition: specificities of eating difficulties children," Physiology \& Behavior, vol. 173, pp. 116-123, 2017.

[11] M. Dsamou, O. Palicki, C. Septier et al., "Salivary protein profiles and sensitivity to the bitter taste of caffeine," Chemical Senses, vol. 37, pp. 87-95, 2012.

[12] T. Cabras, M. Melis, M. Castagnola et al., "Responsiveness to 6-n-propylthiouracil (PROP) is associated with salivary levels of two specific basic proline-rich proteins in humans," PLOS One, vol. 7, Article ID e30962, 2012.

[13] L. Rodrigues, G. da Costa, C. Cordeiro, C. C. Pinheiro, F. Amado, and E. Lamy, "Relationship between saliva protein composition and 6-n-Propylthiouracil bitter taste responsiveness in young adults," Journal of Sensory Studies, vol. 32, 2017.

[14] E. Lamy, G. da Costa, R. Santos et al., "Sheep and goat saliva proteome analysis: a useful tool for ingestive behavior research?" Physiology \& Behavior, vol. 98, pp. 393-401, 2009.

[15] E. Sales Baptista, E. Lamy, M. Mau, F. Capela e Silva, and A. V. Coelho, "Variation in salivary protein composition related to feeding behavior and its ecological implications," 2013.

[16] E. Lamy, G. Graça, G. da Costa et al., "Changes in mouse whole saliva soluble proteome induced by tannin-enriched diet," Proteome Science, vol. 8, p. 65, 2010.

[17] E. Lamy, E. S. Baptista, A. V. Coelho, and F. C. Silva, "Morphological alterations in salivary glands of mice (Mus musculus) submitted to tannin enriched diets: comparison with sialotrophic effects of sympathetic agonists stimulation," The Arquivo Brasileiro de Medicina Veterinária e Zootecnia, vol. $62,2010$.

[18] G. da Costa, E. Lamy, F. Capela e Silva, J. Andersen, E. Sales Baptista, and A. V Coelho, "Salivary amylase induction by tannin-enriched diets as a possible countermeasure against tannins," Journal of Chemical Ecology, vol. 34, pp. 376-387, 2008.

[19] C. Dinnella, A. Recchia, S. Vincenzi, H. Tuorila, and E. Monteleone, "Temporary modification of salivary protein profile and individual responses to repeated phenolic astringent stimuli," Chemical Senses, vol. 35, pp. 75-85, 2010.
[20] A. L. Mandel, C. Peyrot des Gachons, K. L. Plank, S. Alarcon, and P. A. S. Breslin, "Individual differences in AMY1 gene copy number, salivary $\alpha$-amylase levels, and the perception of oral starch," PLoS One, vol. 5, Article ID e13352, 2010.

[21] J. L. Santos, E. Saus, S. V Smalley et al., "Copy number polymorphism of the salivary amylase gene: implications in human nutrition research," Journal of Nutrigenetics and Nutrigenomics, vol. 5, pp. 117-131, 2012.

[22] L. Carreira, P. Midori Castelo, C. Simões, F. Capela e Silva, C. Viegas, and E. Lamy, "Changes in salivary proteome in response to bread odour," Nutrients, vol. 12, p. 1002, 2020.

[23] E. Lamy, V. Santos, S. Barrambana et al., Saliva Protein Composition Relates with Interindividual Variations in Bread Sensory Ratings, Starch - Stärke, Wiley, Hoboken, NJ, USA, 2020.

[24] A. Tvarijonaviciute, N. Martinez-Lozano, R. Rios, M. C. Marcilla de Teruel, M. Garaulet, and J. J. Cerón, "Saliva as a non-invasive tool for assessment of metabolic and inflammatory biomarkers in children," Clinical Nutrition, vol. 39, no. 8, pp. 2471-2478, 2019.

[25] L. Rodrigues, R. Espanca, A. R. Costa et al., "Comparison of salivary proteome of children with different sensitivities for bitter and sweet tastes: association with body mass index," International Journal of Obesity, vol. 43, no. 4, pp. 701-712, 2019.

[26] B. Nunes, M. Barreto, A. P. Gil et al., "The first Portuguese National Health Examination Survey (2015): design, planning and implementation," Journal of Public Health, vol. 41, 2019.

[27] WHO, "Preventing and Managing the Global Epidemic. World Health Organization: Technical Report Series," WHO, Geneva, Switzerland, 2000.

[28] C. Lopes, A. Aro, A. Azevedo, E. Ramos, and H. Barros, "Intake and adipose tissue composition of fatty acids and risk of myocardial infarction in a male Portuguese community sample," Journal of the American Dietetic Association, vol. 107, pp. 276-286, 2007.

[29] P. Moreira, S. Santos, P. Padrão et al., "Food patterns according to Sociodemographics, physical activity, sleeping and obesity in Portuguese children," International Journal of Environmental Research and Public Health, vol. 7, no. 3, pp. 1121-1138, 2010.

[30] E. Lamy, C. Simões, L. Rodrigues et al., "Changes in the salivary protein profile of morbidly obese women either previously subjected to bariatric surgery or not," Journal of Physiology and Biochemistry, vol. 71, 2015.

[31] H. F. Kaiser, "An index of factorial simplicity," Psychometrika, vol. 39, pp. 31-36, 1974.

[32] H. F. Kaiser, "The application of electronic computers to factor Analysis," Educational and Psychological Measurement, vol. 20, pp. 141-151, 1960.

[33] M. D. Contreras-Aguilar, D. Escribano, S. Martínez-Subiela, S. Martínez-Miró, J. J. Cerón, and F. Tecles, "Changes in alpha-amylase activity, concentration and isoforms in pigs after an experimental acute stress model: an exploratory study," BMC Veterinary Research, vol. 14, p. 256, 2018.

[34] E. Lamy, H. Rawel, F. J. Schweigert et al., "The effect of tannins on mediterranean ruminant ingestive behavior: the role of the oral cavity," Molecules, vol. 16, 2011.

[35] L. E. Martin, L. V. Nikonova, K. Kay, A. B. Paedae, R. J. Contreras, and A. M. Torregrossa, "Salivary proteins alter taste-guided behaviors and taste nerve signaling in rat," Physiology \& Behavior, vol. 184, pp. 150-161, 2018. 
[36] S. Soares, N. Mateus, and V. de Freitas, "Interaction of different classes of salivary proteins with food tannins," Food Research International, vol. 49, pp. 807-813, 2012.

[37] L. E. Martin, L. V. Nikonova, K. E. Kay, and A. M. Torregrossa, "Altering salivary protein profile can increase acceptance of a novel bitter diet," Appetite, vol. 136, pp. 8-17, 2019.

[38] C. R. Crawford and C. A. Running, "Addition of chocolate milk to diet corresponds to protein concentration changes in human saliva," Physiology \& Behavior, vol. 225, 2020.

[39] L. Rodrigues, R. Espanca, A. R. Costa et al., "Association between salivary leptin levels and taste perception in children," Journal of Nutrition and Metabolism, vol. 2017, 2017.

[40] L. Rodrigues, G. Costa, C. Cordeiro, C. Pinheiro, F. Amado, and E. Lamy, "Salivary proteome and glucose levels are related with sweet taste sensitivity in young adults," Food \& Nutrition Research, vol. 61, Article ID 1389208, 2017.

[41] L. Franco-Martínez, J. M. González-Hernández, A. Horvatić et al., "Differences on salivary proteome at rest and in response to an acute exercise in men and women: a pilot study," Journal of Proteomics, vol. 214, Article ID 103629, 2020.

[42] A. C. Mosca, M. Stieger, E. Neyraud, H. Brignot, A. van de Wiel, and J. Chen, "How are macronutrient intake, BMI, ethnicity, age, and gender related to the composition of unstimulated saliva? a case study," Journal of Texture Studies, vol. 50, no. 1, 2019.

[43] A. Skinner, Z. Toumpakari, C. Stone, and L. Johnson, "Future directions for integrative objective assessment of eating using wearable sensing Technology," Frontiers in Nutrition, vol. 7, 2020.

[44] C. V. Esteves, W. G. de Campos, M. M. de Souza, S. V. Lourenço, W. L. Siqueira, and C. A. Lemos-Júnior, "Diagnostic potential of saliva proteome analysis: a review and guide to clinical practice," Brazilian Oral Research, vol. 33, p. e043, 2019.

[45] F. M. L. Amado, R. P. Ferreira, and R. Vitorino, "One decade of salivary proteomics: current approaches and outstanding challenges," Clinical Biochemistry, vol. 46, pp. 506-517, 2013. 